\title{
Starburst galaxies and the X-ray background
}

\author{
M. Persic ${ }^{1}$ and Y. Rephaeli ${ }^{2,3}$ \\ 1 INAF/Osservatorio Astronomico di Trieste, via G.B. Tiepolo 11, 34131 Trieste, Italy \\ 2 School of Physics and Astronomy, Tel Aviv University, Tel Aviv 69978, Israel \\ 3 CASS, University of California, San Diego, La Jolla, CA 92093, USA
}

Received 5 September 2002 / Accepted 14 November 2002

\begin{abstract}
Integrated X-ray spectra of an evolving population of starburst galaxies (SBGs) are determined based on the observed spectra of local SBGs. In addition to emission from hot gas and binary systems, our model SBG spectrum includes a nonthermal component from Compton scattering of relativistic electrons by the intense ambient far-IR and the (steeply evolving) CMB radiation fields. We use these integrated spectra to calculate the levels of contribution of SBGs to the cosmic X-ray background assuming that their density evolves as $(1+z)^{q}$ up to a maximal redshift of 5. We find that at energies $\epsilon \lesssim 10 \mathrm{keV}$ this contribution is at a level of few percent for $q \leq 3$, and in the range of $5 \%-15 \%$ for $q \simeq 4.5$. The Compton component is predicted to be the main SBG emission at high energies, and its relative contribution gets progressively higher for increasing redshift.
\end{abstract}

Key words. X-ray: galaxies - galaxies: spiral - stars: formation

\section{Introduction}

In starburst galaxies (SBGs) enhanced star formation activity (lasting typically $\lesssim 10^{8} \mathrm{yr}$ ) drives a chain of coupled stellar and interstellar phenomena that are manifested by intense farinfrared (FIR) emission. The SBG spectrum shows a distinctive large bump of thermal FIR dust emission which is correlated with optical emission (e.g., Silva et al. 1998), a result of the fact that dust emission is reprocessed starlight from a population of hot OB stars. Such stars are short-lived $\left(10^{6-7}\right.$ years $)$ and end up as supernovae. The subsequent radio emission from synchrotron radiation in supernova remnants (SNRs) is tightly correlated with the IR emission (e.g., Condon 1992). Interest in SBGs stems also from the realization that these resemble young galaxies in the earlier universe. Indeed, a starburst phase was very common then, as a result of both astrophysical processes (baryonic infall and early star formation) and dynamical processes (close encounters and mergers which, in turn, trigger star formation). Consequently, the cosmic star formation rate (SFR) was substantially higher at epochs corresponding to $z \gtrsim 1$, with the SFR having either a peak at $1 \lesssim z \lesssim 2$ (Madau et al. 1996), or a plateau out to $z \sim 4$ (Thompson et al. 2001).

A primary manifestation of the starburst activity is X-ray emission. Given the greatly enhanced SFR, energetic phenomena related to stellar evolution - OB stars, X-ray binaries, SNRs, galactic winds, and Compton scattering of ambient FIR and CMB photons off relativistic electrons that are accelerated by $\mathrm{SN}$ shocks - clearly suggest that SBGs are typically more

Send offprint requests to: M. Persic, e-mail: persic@ts.astro.it powerful X-ray emitters than normal galaxies of comparable stellar mass (e.g., Rephaeli et al. 1991; Schmitt et al. 1997). The mean X-ray spectrum of SBGs reflects the diverse nature of high energy activity in these galaxies (Persic \& Rephaeli 2002; Rephaeli et al. 1991, 1995).

The link between SBGs and the cosmic X-ray background (CXB) was made early on (Bookbinder et al. 1980; Stewart et al. 1982; Weedman 1987; Griffiths \& Padovani 1990; Rephaeli et al. 1991, 1995; David et al. 1982; Ricker \& Meszaros 1993; Moran et al. 1999). Most previous estimates of the SBG contribution to the CXB were essentially based on the SBG emission at $2 \mathrm{keV}$, with either no source evolution (Weedman 1987), or with some assumed degree of evolution (Griffiths \& Padovani 1990; Ricker \& Meszaros 1993). Clearly, the use of the $2 \mathrm{keV}$ emission as baseline is unjustified given the wide spectral range (3-100 keV) over which the CXB seems to have been well determined (e.g., Gruber et al. 1999). The SBG contribution was also evaluated based on the emission over a wider spectral range, either in the context of an assumed spectral model (Ricker \& Meszaros 1993), or from a statistically deduced mean spectrum (determined from the HEAO-1 A2+A4 datasets: Rephaeli et al. 1991, 1995).

In this paper we compute the contribution of SBGs to the CXB incorporating recent results in the study of SBGs. In particular, we use the knowledge of SBG spectral properties gained from direct ASCA, BeppoSAX, and RXTE observations of local galaxies. This, complemented with an assumed form for the cosmic evolution of the SBG X-ray luminosity density, enables us to compute the component of the CXB 
spectrum which originates in SBGs. We first (Sect. 2) review the spectral properties of (local) SBGs, and proceed to discuss the local SBG luminosity density and its cosmic evolution in Sects. 3 and 4. The predicted CXB spectrum is then calculated in Sect. 5, followed by a discussion (Sect. 6), and a summary (Sect. 7). Our calculations are carried out in the context of the Einstein-de Sitter $\left(\Omega_{\mathrm{m}}=1, \Omega_{\Lambda}=0\right.$; hereafter EdS) and (currently favoured) flat $\Lambda\left(\Omega_{\mathrm{m}}=0.3, \Omega_{\Lambda}=0.7\right)$ cosmological models, with $H_{0}=50 \mathrm{~km} \mathrm{~s}^{-1} \mathrm{Mpc}^{-1}$.

\section{X-ray spectral properties of SBGs}

Local SBGs have been repeatedly observed in X-rays (see Fabbiano 1989; Rephaeli et al. 1995; Dahlem et al. 1998; Persic \& Rephaeli 2002; and references therein). In most of these $(\sim 10)$ galaxies the $0.5-10 \mathrm{keV}$ spectra can be well fit by a combination of thermal, low-temperature $(k T \simeq 0.8 \mathrm{keV})$ emission, and photoelectrically self-absorbed emission that can be represented by an exponentially cutoff power law (CPL) of the form $x^{-\Gamma} \mathrm{e}^{-x / k T}$, where $x$ is the energy in the source frame, $\Gamma$ is the photon index and $k T$ is the cutoff energy ${ }^{1}$ (Persic \& Rephaeli 2002; Persic et al. 2002; see Fig. 1).

The low-temperature and CPL components can be interpreted as due to emission from galactic winds and X-ray binaries, respectively. From a detailed discussion of a synthetic X-ray spectrum of SBGs, based on an evolutionary model of galactic stellar populations and on the X-ray spectra of the relevant emission processes, Persic \& Rephaeli (2002) have suggested that X-ray binaries contribute most of the $2-15 \mathrm{keV}$ emission. Both types of X-ray binaries, high- and low-mass systems, have spectra that can be described as variously cutoff power laws (White et al. 1983; Christian \& Swank 1997). In general, the population-averaged spectrum emitted by a realistic mix of X-ray binaries (high- and low-mass systems of various luminosities in Galactic proportions) can be described as a CPL with $\Gamma \sim 1.2$ and $k T \sim 8 \mathrm{keV}$ (Persic \& Rephaeli 2002).

The enhanced SFR activity in SBGs results in a high SN rate and consequently - among other effects - in a higher rate of electron acceleration to relativistic energies. This prospect, coupled with the higher energy density in the enhanced FIR radiation field, almost certainly implies that Compton scattering of the electrons by both the FIR and CMB fields yields a substantially higher level of nonthermal X-ray emission than in normal galaxies (Schaaf et al. 1989; Rephaeli et al. 1991). A quantitative assessment of this process in NGC 253 was given by Goldshmidt \& Rephaeli (1995). Substantial PL X-ray emission may also be produced in the galactic nucleus, especially

\footnotetext{
${ }^{1}$ Earlier spectral studies, in which simple thermal and PL models were fitted to the main emission component, concluded that $\gtrsim 5 \mathrm{keV}$ thermal or $\Gamma \sim 2$ PL fits were similarly successful for all analyzed objects (NGC 253, M 82: Ptak et al. 1997, Cappi et al. 1999; M 83: Okada et al. 1997; NGC 1569: Della Ceca et al. 1996; NGC 2146: Della Ceca et al. 1999; NGC 2903: Mizuno et al. 1998; NGC 3256: Moran et al. 1999; NGC 3310, NGC 3690: Zezas et al. 1998), and hence they were unable to reach definitive conclusions on the nature of the main component.
}

if the SB-driven turbulence of the gas increases the mass inflow rate onto a central black hole (e.g., Veilleux 2001). Such spectral components can be described as $\propto x^{-\alpha}$ with the photon index in the range $\alpha \sim 1.6-1.8$ over a broad energy range (e.g., Goldshmidt \& Rephaeli 1995; Rothschild et al. 1983).

The spectral similarity shown by the local SBGs (see also Roberts et al. 2001) provides a reasonably good basis for a first approximation at modelling the population as a whole. We therefore assume that a typical starburst spectrum consists of a $0.8 \mathrm{keV}$ thermal component (i.e., a thermal bremsstrahlung spectrum, with the Gaunt factor numerically calculated as prescribed by Itoh et al. 2000), plus a CPL with photon spectral index $\Gamma=1.2$ and cutoff energy $k T=7.5 \mathrm{keV}$. This second component is photoelectrically absorbed in situ through a HI gas of column density $N_{\mathrm{H}}$. The two components are normalized such that the thermal-to-CPL energy flux ratio in the $2-10 \mathrm{keV}$ band is 0.03 .

In addition to the wind and binary emission, we include also two emission components originating from Compton scattering of relativistic electrons by the FIR and CMB radiation fields. While the full calculation of the respective fluxes requires a detailed, self-consistent solution of the kinetic equation for the electrons (taking into account their varius energy loss mechanisms and their propagation mode in the disk and halo - see, e.g., Rephaeli 1979), we greatly simplify the treatment by keeping only the most salient features pertinent to our discussion here. Assuming the typical value of 0.8 for the radio (spectral energy) index of the radio synchrotron flux in the disk, and estimating typical energy loss times of the electrons by synchrotron emission and Compton scattering off the FIR and $\mathrm{CMB}$ radiation fields, we can roughly represent the latter two Compton components as follows: A primarily inner-disk FIR-scattered component with a (photon) flux that is $\propto x^{-1.8}$, and a (primarily) outer-disk and halo CMB-scattered component $\propto x^{-2.3}$ due to electrons from the higher-energy region of the (electron) spectrum that is steepened by radiative losses.

The relative contribution of the Compton fluxes is substantially uncertain since this emission has not yet been unequivocally detected in SBGs (see, e.g., Goldshmidt \& Rephaeli 1995; Rephaeli \& Gruber 2002). However, the expectation that Compton scattering of the enhanced relativistic electron population by the intense ambient FIR radiation field provides strong motivation for inclusion of this emission in our modelling of SBG X-ray emission. Our modelling of the Compton fluxes is based on the radio properties of the nearby SBGs M 82 and NGC 253, and well known Compton-synchrotron relations (e.g., Tucker 1975) between the radio luminosity and deduced mean magnetic field in the disk (typically, $\sim 10 \mu \mathrm{G}$, but with appreciable uncertainty) and the predicted X-ray flux from Compton scattering of the radio producing electrons by the FIR and CMB fields. The relative strength of these two radiation fields largely determines (under typical conditions in local SBGs) the normalization between their respective fluxes. The energy density of the FIR radiation field, $U_{\text {fir }}$, was estimated by Goldshmidt \& Rephaeli (1995) in the context of a quantitative model for the emission from warm dust. We adopt their result, $U_{\text {fir }} \simeq 10 U_{0}$ where $U_{0}$ is the energy density in the $\mathrm{CMB}$, as roughly typical in the inner region of a SBG disk. 
Doing so, and using the usual Compton-synchrotron relations, we obtain that in local SBGs we might expect that the FIR and CMB Compton contributions to the overall 2-10 keV (energy) flux are roughly at a level of $5 \%$ and $0.5 \%$, respectively. We do not yet have unequivocal evidence for nonthermal Compton Xray emission in SBGs. Power-law fits to both ASCA and RXTE data on M 82 and NGC 253 were found to be formally acceptable (e.g. Matsumoto \& Tsuru 1999; Rephaeli \& Gruber 2002), but a closer assessment of the residuals of such fits over the wider energy range afforded by RXTE leads only to what are essentially upper limits to nonthermal contribution. With the higher spatial resolution of the Chandra satellite it was clearly established that some of the $2-10 \mathrm{keV}$ emission in M 82 is diffuse, emanating from a central elliptical region, $0.8 \mathrm{kpc} \times 0.8 \mathrm{kpc}$, that is coplanar and coaligned with the galactic disk (Griffith et al. 2000). According to these authors the spectral fits to these measurements make a Compton origin of most of the emission quite likely. The luminosity of the extended emission region is $L_{2-10} \simeq 2.2 \times 10^{39} \mathrm{erg} \mathrm{s}^{-1}$ (for an assumed distance $D=3.6 \mathrm{Mpc}$ ); this value constitutes $\sim 5 \%$ of the total $2-10 \mathrm{keV}$ luminosity $\left(L_{2-10} \simeq 4.5 \times 10^{40} \mathrm{erg} \mathrm{s}^{-1}\right)$ measured by BeppoSAX (Persic \& Rephaeli 2002). Given that some or most of this diffuse hard component in M 82 is thermal as suggested by the presence of a substantial $6.7 \mathrm{keV}$ Fe-K emission, the figure of $5 \%$ is actually a strong upper limit on the Compton contribution in $\mathrm{M} 82^{2}$.

Finally, let $f(x)$ be the spectral profile (in the source frame), normalized in the band relative to which the surface brightness is being computed. In accord with our discussion above, the $\mathrm{X}$-ray photon spectral profile of a SBG is a superposition of components that can be written as

$$
\begin{aligned}
f(x)= & \beta f_{\mathrm{TB}}(x)+(1-\beta) \mathrm{e}^{-N_{\mathrm{H}} \sigma_{\mathrm{pa}}(x)} f_{\mathrm{cPL}}(x) \\
& +\eta_{1} \mathrm{e}^{-N_{\mathrm{H}} \sigma_{\mathrm{pa}}(x)} f_{\mathrm{C}}^{\mathrm{FIR}}(x)+\eta_{2} f_{\mathrm{C}}^{\mathrm{CMB}}(x, z)
\end{aligned}
$$

where $x \equiv \epsilon(1+z)$ is the energy (in $\mathrm{keV}$ ) in the source frame (and $z$ is the redshift; $\epsilon$ is the energy in the observer's frame), $\sigma_{p a}$ is the cross section for photoelectric absorption per unit hydrogen column density $N_{\mathrm{H}}$ within the source (in the calculations we have adopted the analytic expression for $\sigma_{\text {pa }}$ given by Morrison \& McCammon 1983; $N_{\mathrm{H}}$ is taken at a nominal level of $10^{22} \mathrm{~cm}^{-2}$ ), and $\beta, \eta_{1}$, and $\eta_{2}$ are constants of normalization that are estimated as described above. Note that the CMB scattered flux is $z$-dependent, an important fact that will be elaborated upon in Sect. 5.

\section{Local luminosity density}

In order to calculate the contribution of SBGs to the CXB we need to model the population at all $z$, beginning - of course with the local source density which can be deduced from the FIR luminosity function. This has been determined from the $60 \mu$ m flux-limited IRAS samples (Saunders et al. 1990),

$\phi(L) \mathrm{d} \log L=C\left(\frac{L}{L^{\star}}\right)^{1-\alpha} \mathrm{e}^{-\frac{1}{2 \sigma^{2}} \log ^{2}\left(1+L / L^{\star}\right)} \mathrm{d} \log L$.

${ }^{2}$ Also for NGC 3256 (the brightest among local SBGs), Compton scattering has been argued to contribute appreciably to the $2-10 \mathrm{keV}$ flux (Moran et al. 1999).
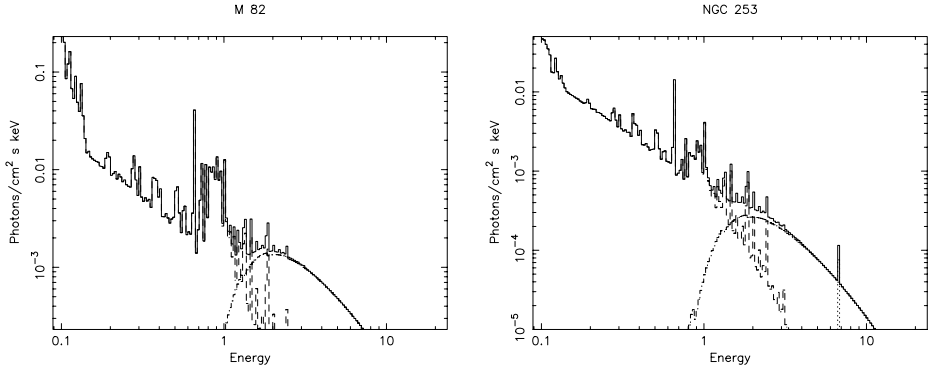

Fig. 1. The best-fit thermal + CPL models for M 82 (left) and NGC253 (right), based on BeppoSAX data (see Persic \& Rephaeli 2002).

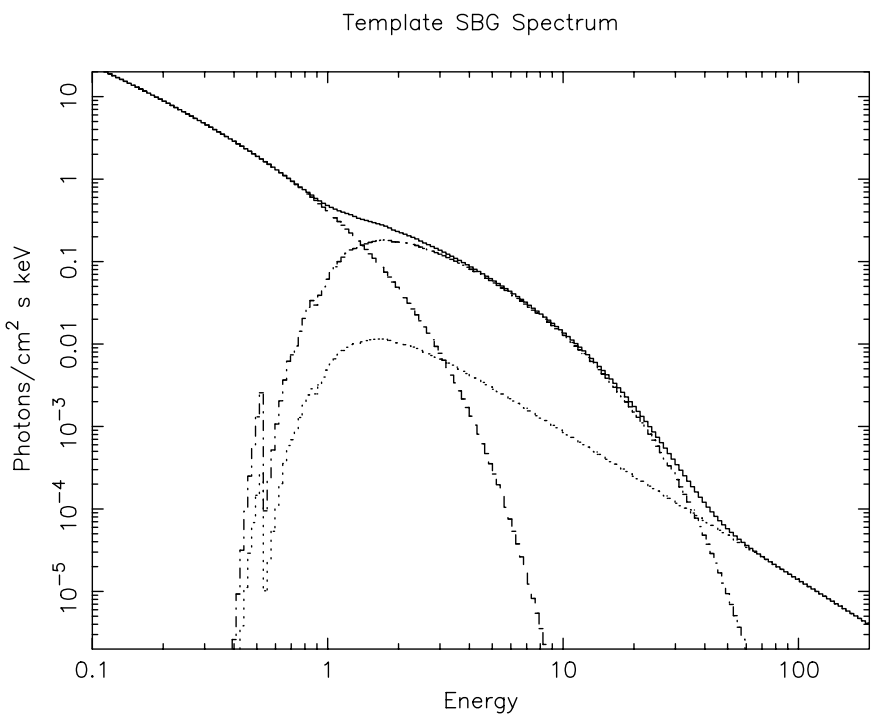

Fig. 2. The template photon SBG spectrum assumed in the present calculations. It consists of: (i) an unabsorbed $0.8 \mathrm{keV}$ thermal bremsstrahlung component, (ii) an exponentially cutoff PL with photon index $\Gamma=1.2$ and cutoff energy $k T=7.5 \mathrm{keV}$, absorbed through $N_{\mathrm{H}}=10^{22} \mathrm{~cm}^{-2}$, and (iii) a similarly absorbed PL with photon in$\operatorname{dex} \alpha_{1}=1.8$. (The much fainter unabsorbed PL with photon index $\alpha_{2}=2.3$ is not shown here.) The flux normalizations are as specified in the text.

Sources in the IRAS Point Source Catalog with fluxes measured in all four IRAS bands and with individual, identifiable galaxy types inhabit distinct and well-defined areas in colorcolor diagrams (Rowan-Robinson \& Crawford 1989). Each one of these distinct components of the IRAS point-source population largely produces most of the emission at specific wavelengths, and is characterized by a specific set of parameters for Eq. (2a). For the warm-source component that peaks at $\sim 60 \mu \mathrm{m}$ which is thought to be attributable to a dusty SBG population, the parameters in Eq. (2a) take the values:

$$
\left\{\begin{array}{l}
C=3.25 \times 10^{-4} \mathrm{Mpc}^{-3} \\
\log L^{\star}=9.99 \\
\alpha=1.27 \\
\sigma=0.626
\end{array}\right.
$$

for luminosities in the range $6 \leq \log \left(L_{60} / L_{\odot}\right) \leq 12.5$. Pearson $\&$ Rowan-Robinson (1996) modeled the (sub-mm, IR, optical) number-flux relation for extragalactic sources by including a SBG population described by a local luminosity function 
as in Eq. (2). The local $60 \mu \mathrm{m}$ luminosity density implied by Eq. (2) is:

$\mathcal{L}_{60 \mu \mathrm{m}}=8.4 \times 10^{6} L_{\odot} \mathrm{Mpc}^{-3}$.

For a sample of local SBGs observed with ASCA, the ratio of $2-10 \mathrm{keV}$ flux to $60 \mu \mathrm{m} \mathrm{flux}^{3}$ is $(4 \pm 1) \times 10^{-4}$. Assuming this to be the mean value for the whole SBG population, we have for the $2-10 \mathrm{keV}$ luminosity density

$\mathcal{L}_{2-10 \mathrm{keV}}(0)=(1.3 \pm 0.3) \times 10^{37} \mathrm{erg} \mathrm{s}^{-1} \mathrm{Mpc}^{-3}$.

Note that in our previous estimates of the SBG contribution to the CXB (Rephaeli et al. 1991, 1995) we did consider only the high end $\left(L_{\mathrm{FIR}} \geq 10^{11} L_{\odot}\right)$ of the FIR luminosity function as representative of the SBG population. Adopting the current more inclusive luminosity function (Pearson \& Rowan-Robinson 1996) allows a more realistic estimate that accounts also for the emission from fainter SBGs. By integrating the FIR luminosity function over the above (logarithmic) range $([6,12.5])$, we compute a density of $\simeq 5.9 \times 10^{-3} \mathrm{Mpc}^{-3}$ for galaxies that are identified here as members of the broader SBG population. (Obviously, this value of the density is much higher than that of the more luminous galaxies, but this difference is largely compensated for by the much lower mean FIR luminosity, $L_{\mathrm{FIR}} \sim 10^{9} L_{\odot}$, of the SBGs that we include in this work.)

\section{Cosmic evolution}

Galactic evolution may generally be reflected in density and luminosity variations over cosmic time due to the continued formation, interaction and merging, and through intrinsic changes of the luminosity due to dynamical and thermal evolution of stars and interstellar gas. The evolution of SBGs is likely to be particularly pronounced as the SB phase is thought to be triggered by galactic interactions and is relatively short lived. These imply that SBGs were more abundant at earlier times than would be expected based solely on pure density evolution of sources in an expanding background $\left(\propto(1+z)^{3}\right)$.

Observationally, the evolution of SBGs is inferred from radio, IR, and optical measurements. At radio frequencies, two distinct populations are identified at $1.4 \mathrm{GHz}$ (Benn et al. 1993): radio-loud, and radio-faint, the first consisting of giant ellipticals and QSOs, and the second mainly of spirals (SBGs and radio-quiet QSOs). This latter faint population is responsible for the low-flux upturn in the differential counts, and is indistinguishable from the SBG-dominated $60 \mu \mathrm{m}$ population (e.g., Danese et al. 1987; Franceschini et al. 1988), which can be fit by a model using pure luminosity evolution of the form $L(z)=L(0)(1+z)^{q}$ with $q=3.1$ (Rowan-Robinson et al. 1993). At IR wavelengths, Lonsdale et al. (1990) concluded that the dominant population in the faint $60 \mu \mathrm{m}$ counts from the

\footnotetext{
3 The objects are: M 82, NGC 253, NGC 2903, NGC 3690. The $60 \mu \mathrm{m}$ fluxes are calculated using the prescription $F(60)=2.58 \times$ $10^{-14} f_{v}(60)$, where $f_{v}$ is the nominal flux densities listed in the IRAS catalog (Lonsdale et al. 1985; see also Shapley et al. 2001). The 2-10 keV fluxes are from Ptak et al. (1997) (M 82, NGC 253), Mizuno et al. (1998) (NGC 2903), Zezas et al. (1998) (NGC 3690).
}

IRAS Faint Source Survey consists of strongly evolving SBGs ${ }^{4}$. Thus, it appears that from $60 \mu \mathrm{m}$ to radio wavelengths what is mainly sampled is starburst activity. SBG evolution, either of the pure luminosity type (Lilly et al. 1995; Ellis et al. 1996; Fried et al. 2001), or of the pure density type (Lin et al. 1999), is deduced also from optical surveys.

The luminosity density of SBGs evolves mainly as result of their steeply varying density, and - to a smaller extent, as discussed below - the changes in the relative strength of the spectral components. Density evolution is represented by the factor $(1+z)^{q}$, with $q \geq 3$ after the process of galaxy formation began in ernest at some early redshift, $z_{\mathrm{m}}$. Since we do not know when exactly galaxies began forming, the modelling the starburst phase of early galaxies necessitates viewing both $q$ and $z_{\mathrm{m}}$ as essentially free parameters ${ }^{5}$. We factor out the dominant redshift dependence of the luminosity density:

$\mathcal{L}(z)=\mathcal{L}(0)(1+z)^{q} \phi(z)$,

with all the spectral dependence on $z$ collected in the function $\phi(z)$; this function will be specified below. The evolution of SBGs is then largely gauged by the index $q^{6}$; an estimate for $\mathcal{L}(0)$ is given in Eq. (4).

\section{SBG emission as a background}

The surface brightness due to a population of unresolved sources is given by (e.g., Boldt 1987)

$I=\frac{1}{4 \pi} \int \mathcal{L} \mathrm{d} \ell$

${ }^{4}$ In ultra-luminous IR galaxies (ULIRGs), which are the high$L_{60 \mu \mathrm{m}}$ tail of the "warm" $60 \mu \mathrm{m}$-selected IRAS population, the starformation activity (Kim \& Sanders 1998a) is very strong; these undergo cosmic evolution (Kim \& Sanders 1998b) similar to the one derived for the whole $60 \mu \mathrm{m}$-selected SBG sample. In addition, a preliminary XMM-Newton study of a sample of ULIRG spectra (Franceschini et al. 2002) suggests properties fairly similar to those of SBG spectra: i.e., the "starburst signature" discussed in Sect. 2, plus sometimes a hard PL component (interpretetd as SB-obscured AGNs). ULIRGs are then implicitly included in the calculations reported in this paper.

5 As an example, we derive an evolution function of the type in Eq. (5) within a toy model of galaxy evolution. The comoving number density of galaxies undergoing a starbursting phase at any given epoch $z, n_{\mathrm{SBG}}(z)$, is closely related to the total galaxy density at that time, $n_{\mathrm{G}}(z)$. If so, we expect the comoving SBG fraction to increase steeply with $z$. The interaction rate between galaxies is $\propto n_{\mathrm{G}}(z)^{2} v$ (with constant interaction cross section), where $n_{\mathrm{G}}(z)$ is the galaxy density, and $v \equiv \sqrt{(\bar{\delta})^{2}}$ is the rms pairwise velocity dispersion of interacting galaxies. Because of the strong $n_{\mathrm{G}} \propto(1+z)^{3}$ dependence we can ignore the relatively weak dependence on $v$. This implies that in a flat universe the volume density of SBGs at any given time is $n_{\mathrm{SBG}}(z)=n_{\mathrm{SBG}}(0)(1+z)^{4.5}$.

${ }^{6}$ Evidence for evolution of the galaxy luminosity density has been found also in other bands. Based on $z \leq 1.5, B$ - and $U V$-selected galaxies, the deduced evolution rate has $q=2.4 \pm 1.0$ for an EdS cosmology and $q=1.7 \pm 1.0$ for a flat $\Lambda$ model (Wilson et al. 2002). Earlier work, based on $z \leq 1, I$-band selected galaxies, claimed $q \lesssim 4$ (Lilly et al. 1996). 

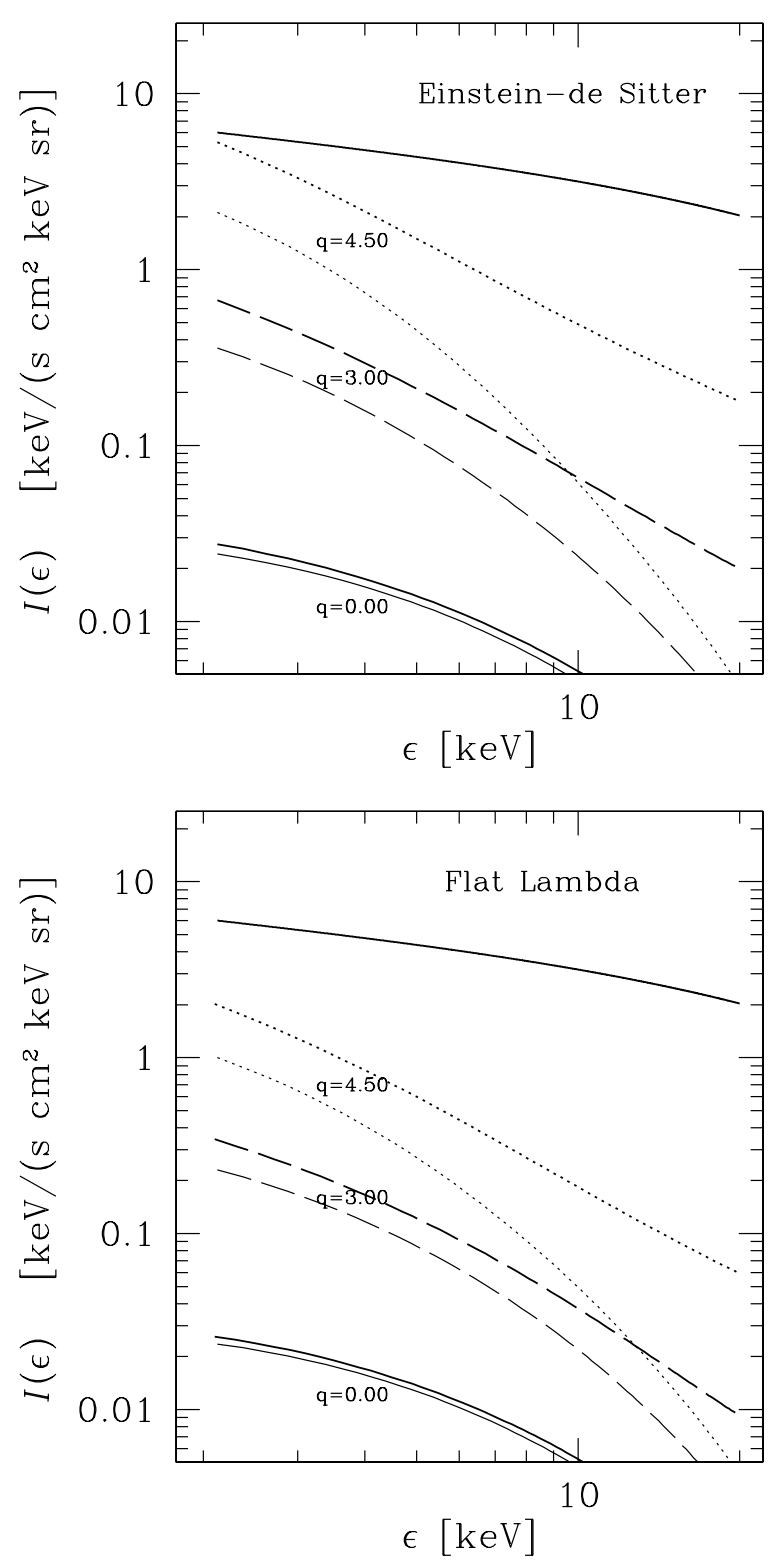

Fig. 3. The spectrum of the integrated background light arising from SBGs evolving out to $z_{\mathrm{m}}=5.0$, for different values of the evolution parameter. For each of the three values $q=0,3,4.5$, indicated by solid, long-dashed, and dotted curves, the lighter curve represents the unabsorbed bremsstrahlung emission plus absorbed CPL, and the heavy curve shows the sum of these two components plus Comptonized emission (which consists of an absorbed $\alpha_{1}=1.8 \mathrm{PL}$ and unabsorbed $\alpha_{2}=2.3 \mathrm{PL}$ ). The local SBG X-ray luminosity density is given in Eq. (4), its cosmic evolution is given in Eq. (5), and the template SBG spectrum is shown in Fig. 2. The solid black curve on top represents the observed CXB (see Eq. (12)).

where $\mathcal{L} \equiv \int L \phi(L) \mathrm{d} L$ is the comoving volume emissivity, and $\mathrm{d} \ell$ is the line element. Since $\mathrm{d} \ell$ is related to the look-back time $\tau$ and the redshift $z$ by $\mathrm{d} \ell=c /(1+z) \mathrm{d} \tau$, we obtain

$I=\frac{c}{4 \pi} \int \frac{\mathcal{L} \mathrm{d} \tau}{1+z}$
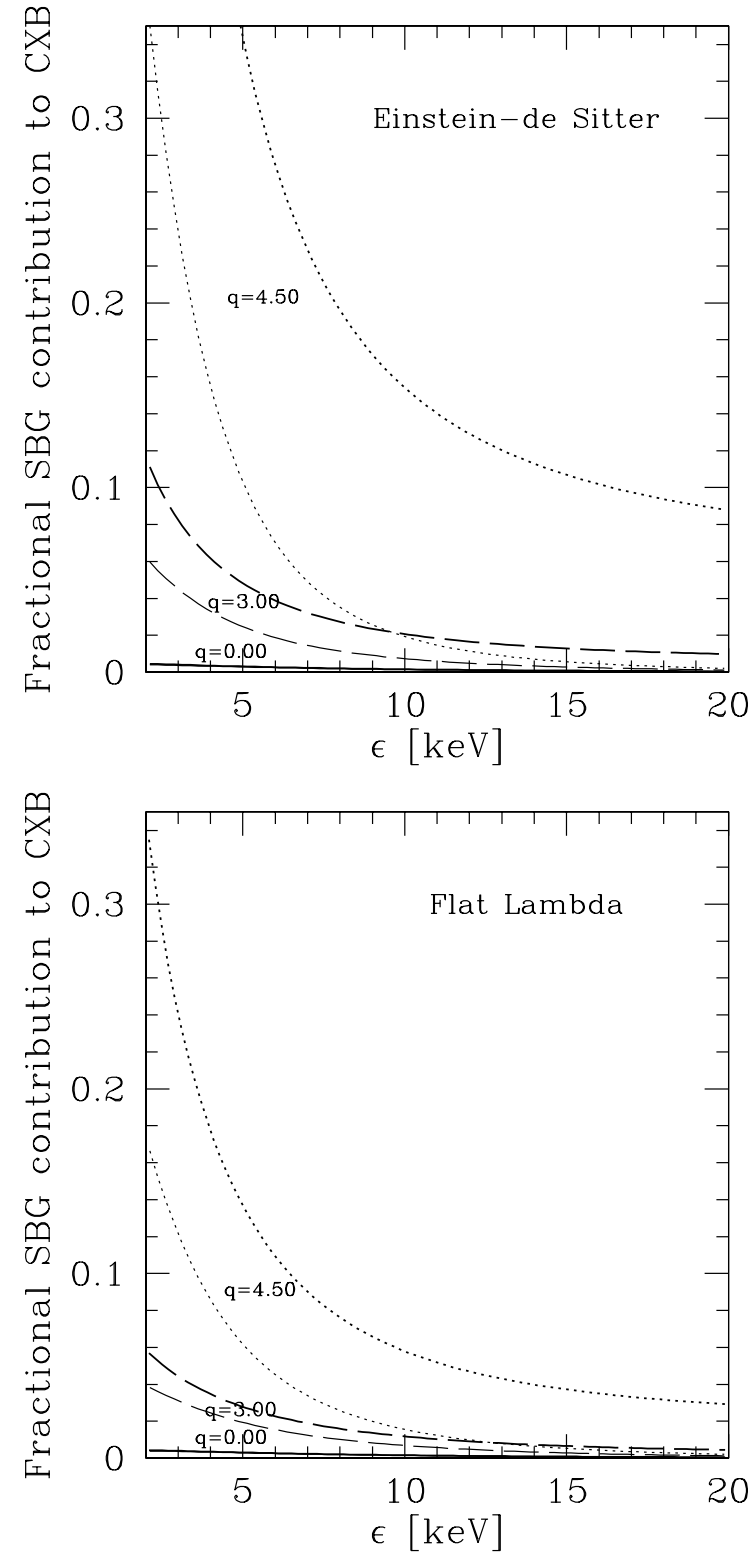

Fig. 4. The fraction of the CXB arising from SBGs as a function of energy, for various strengths of cosmological evolution. Models, parameter values, and symbols are as in Fig. 3 .

The ensuing spectral density is then given by

$\frac{\mathrm{d} I}{\mathrm{~d} \epsilon}=\frac{c}{4 \pi} \int \frac{\mathrm{d} \mathcal{L}}{\mathrm{d} \epsilon_{0}} \mathrm{~d} \tau$

where $\epsilon$ is the energy measured in the observer frame and $\epsilon_{0}=$ $\epsilon(1+z)$ the energy measured in source rest frame.

The look-back time $\tau$ is related to the cosmic time $t$ by $\tau \equiv$ $t_{0}-t$ (with $t_{0}$ the present age of the universe), and $t$ is related to the redshift $z$ by

$$
\begin{aligned}
\frac{\mathrm{d} t}{\mathrm{~d} z}= & -\frac{1}{1+z} \frac{1}{H_{0}} \\
& \times \sqrt{\frac{1}{\Omega_{\mathrm{r}}(1+z)^{4}+\Omega_{\mathrm{m}}(1+z)^{3}+\Omega_{\Lambda}+\left(1-\Omega_{0}\right)(1+z)^{2}}}
\end{aligned}
$$

where $\Omega_{\mathrm{r}}, \Omega_{\mathrm{m}}, \Omega_{\Lambda}$, and $\Omega_{0}$ are the current density parameters for radiation, matter, $\Lambda$, and their sum, respectively. In the 
matter dominated era,

$$
\frac{\mathrm{d} \tau}{\mathrm{d} z}=\frac{1}{H_{0}} \times\left\{\begin{array}{ll}
(1+z)^{-2.5} & \text { EdS } \\
{\left[(1+z) \sqrt{0.3(1+z)^{3}+0.7}\right]^{-1}} & \text { flat } \Lambda
\end{array} .\right.
$$

The SBG photon spectrum we have used in our calculations is given in Eq. (1). Whereas the first two spectral components in Eq. (1) have been adequately discussed in many previous works, the Compton CMB component needs to be further elaborated upon here because of its enhanced role in early SBGs. Most of the X-ray emission from a SBG is triggered by stellar activity and involves the internal properties of the galaxy during a starburst phase of its evolution. As such, the emission is largely independent of redshift, if the main characteristics of this phase have not changed over cosmic time. The Compton component of the emission has evolved significantly, however, due to the much higher energy density of the CMB at $z>1$. As we have noted in Sect. 2, in a local SBG the FIR energy density is typically much higher than that of the CMB, by as much as a factor of $\sim 10$ (Goldshmidt \& Rephaeli 1995), so the Compton CMB component begins to dominate already at $\sim 1$. This, and the fast evolving SBG density, result in a very steep combined spectral and density evolution of the emission from SBGs at high X-ray energies where Compton scattering is the main emission component in SBGs. Due to the steep $(1+z)^{4}$ dependence of the CMB energy density, $f_{\mathrm{C}}^{\mathrm{CMB}}(x, z)$ has an explicit dependence on $z$ in addition to the implicit dependence through $x$.

With $\mathcal{L}\left(\epsilon_{0}, z\right)=\mathcal{L}(z) \phi\left(\epsilon_{0}\right)$, where $\mathcal{L}(z)$ is given in Eq. (5) and $\phi\left(\epsilon_{0}\right) \propto \epsilon_{0} f\left(\epsilon_{0}\right)$ is the SBG energy spectrum (source frame) normalized by the $2-10 \mathrm{keV}$ flux, we have for the total intensity at an observed energy $\epsilon$ due to a population of SBGs undergoing cosmic evolution according to Eq. (5) out to a maximal redshift $z_{\mathrm{m}}$

$I(\epsilon)=\frac{c \mathcal{L}(0)}{4 \pi H_{0}} \cdot\left\{\begin{array}{ll}\int_{0}^{z_{\mathrm{m}}} \frac{(1+z)^{q} \phi[\epsilon(1+z), z]}{(1+z)^{2.5}} \mathrm{~d} z & \text { EdS } \\ \int_{0}^{z_{\mathrm{m}}} \frac{(1+z)^{q} \phi[\epsilon(1+z), z]}{(1+z) \sqrt{0.3(1+z)^{3}+0.7}} \mathrm{~d} z & \text { flat } \Lambda\end{array}\right.$.

The integrated intensity from SBGs was calculated at (the source frame) energies $\epsilon_{0}=2-120 \mathrm{keV}$, for values of the evolutionary index $q$ in the range $0.0 \leq q \leq 4.5$, and adopting $z_{\mathrm{m}}=5$. In Fig. 3 we show the integrated background light arising from an evolving population of SBGs as a function of the (observed) energy, $\epsilon$. To determine the fractional contribution of SBGs to the CXB intensity in the $2-20 \mathrm{keV}$ band, for the latter we use the analytic fit:

$I_{\mathrm{CXB}}(\epsilon)=7.877 \epsilon^{-0.29} \mathrm{e}^{-\epsilon / 41.13}$,

with $I_{\mathrm{CXB}}$ measured in $\mathrm{keV} /\left(\mathrm{cm}^{2} \mathrm{~s} \mathrm{keV} \mathrm{sr}\right)$ and $\epsilon$ measured in $\mathrm{keV}$, given by Gruber et al. (1999) in the 3-60 keV band (based on $H E A O-1 / \mathrm{A} 2+\mathrm{A} 4$ all-sky data: dominated by $\mathrm{A} 2$ proportional-counter data for $\epsilon<15 \mathrm{keV}$, and by $\mathrm{A} 4$ scintillator-experiment data for $\epsilon>30 \mathrm{keV}$ ) and shown by Gendreau et al. (1995) and Chen et al. (1997) to hold down to $1 \mathrm{keV}$ (from ASCA data, based on a composite sample of the sky corresponding to less than a square degree and therefore limited by by sky surface brightness fluctuations) ${ }^{7}$. In Fig. 4 we plot the SBG fractional contribution to the CXB as a function of (observed) energy.

Looking at Fig. 3 we see that for energies $\epsilon \gtrsim 5 \mathrm{keV}$ the shape of the SBG background light gets progressively steeper than that of the $\mathrm{CXB}$, for the range of values of $q$ considered here. (The steep rise of the Comptonized CMB contribution moderates the decline somewhat.) From Fig. 4 it is seen that for no cosmic evolution $(q=0)$ the contribution of SBGs to the CXB is $<1 \%$ at all energies; while for an evolution as steep as the maximum permitted by IR data $(q \simeq 3)$ the contribution is $\sim 5 \%$ at $5 \mathrm{keV}, \sim 2 \%$ at $10 \mathrm{keV}, \sim 1 \%$ at $15 \mathrm{keV}$, and vanishing at higher energies. This is so for the EdS model; the corresponding results are even lower for the flat $\Lambda$ model.

Also of interest is to determine the $z$ dependence of the superposed SBG emission (at a given energy) in order to assess when the contribution is maximal. This can be readily found from the integrand of the $z$-integrated spectral intensity in Eq. (10). Representing the main part of the spectral energy flux of a SBG as $\phi\left(\epsilon_{0}\right) \propto \epsilon_{0} f\left(\epsilon_{0}\right) \propto \epsilon_{0}^{1-\Gamma}$, with $\Gamma \sim 2$ in the 1-200 keV band (see Fig. 2), we see that in the EdS model the integrand in Eq. (10) is $\propto(1+z)^{q-1.5-\Gamma}$. Thus, in evolving models the relative contribution of SBGs increases with $z$ if $q>3.5$, and decreases if $q<3.5$. While the critical value of $q$ for which this transition occurs is different in the flat- $\Lambda$ model, the behavior is qualitatively similar. Note that, as emphasized already, the contribution of the Compton component of the flux grows much more steeply due to the fact that the CMB energy density is $\propto(1+z)^{4}$.

\section{Discussion}

The starting point in our treatment and previous similar works is the identification of SBGs based on their spectral properties at $60 \mu \mathrm{m}$, particularly the use of the same estimate for the local $60 \mu \mathrm{m}$ luminosity density, $\mathcal{L}_{60 \mu \mathrm{m}}$. However, most previous studies were limited to the consideration of only the low-energy ( $\leq 2 \mathrm{keV}$ ) contribution of SBGs to the CXB. Weedman (1987), Griffiths \& Padovani (1990), and Ricker \& Meszaros (1993), having based their estimates on Einstein data, evaluated the SBG contribution at $2 \mathrm{keV}$. More recently, Moran et al. (1999)

${ }^{7}$ Although the spectral shape of the CXB is very well established, there is some question about the normalization, especially at $\epsilon \lesssim 3 \mathrm{keV}$. Under the assumption that Gruber et al.'s (1999) composite multi-experiment fit (which in the $3-50 \mathrm{keV}$ band is very close to the spectrum implied by the HEAO-1/A2 data alone (Marshall et al. 1980; Boldt 1987)) is valid down to $1 \mathrm{keV}$, we note that the $H E A O-1$ spectrum is uniformly lower, by $\sim 11 \%$, than the PL fit to the ASCA/SIS spectrum, $I_{\mathrm{CXB}}(\epsilon)=8.6 \epsilon^{-0.33} \mathrm{keV} /\left(\mathrm{cm}^{2} \mathrm{~s} \mathrm{keV} \mathrm{sr}\right)$ (Gendreau et al. 1995). Actually, the discrepancy could be even worse, were the HEAO-1/A2 result to be renormalized downwards to account for the fact that it includes relatively bright unresolved sources that would have been resolved by ASCA. In addition, the ROSAT observed CXB (at $\epsilon>1 \mathrm{keV}$ ), though limited by surface brightness fluctuations estimated at $\sim 10 \%$ (Miyaji et al. 1998), suggests a CXB normalization $\gtrsim 20 \%$ higher than that obtained from the HEAO-1/A2 result. Given this state of affairs, we assume that a smooth extrapolation of Gruber et al.'s (1999) HEAO-1-based formula down to $2 \mathrm{keV}$ is safe enough for our current purposes. 
estimated the SBG contribution to the $5 \mathrm{keV}$ CXB from ASCA data, based on the assumption that the $5 \mathrm{GHz}$ radio emission from galaxies lacking a radio-loud AGN can serve as a gauge of star-formation activity, and that the value measured in NGC 3256 for the $5 \mathrm{keV}$ to $5 \mathrm{GHz}$ flux ratio is universal. They used the $5 \mathrm{GHz}$ number-count-flux relation, $\log N-\log S_{5 \mathrm{GHz}}$, in the sub-mJy regime, and estimated that SBGs contribute $\sim 12 \%-28 \%$ of the $5 \mathrm{keV}$ CXB. A less limited assessment of the SBG contribution was implemented (Rephaeli et al. 1991, 1995 ) by considering the SBG emission in the $2-30 \mathrm{keV}$ band. However, due to lack of observational data, our previous works were based on a very preliminary statistical analysis of stacked (low-exposure HEAO-1 A2+A4) data. Here, on the other hand, we have estimated the $2-10 \mathrm{keV}$ luminosity density using the detailed $60 \mu \mathrm{m}$ and 2-10 keV luminosities measured for essentially the same sample of local SBGs whose X-ray spectra have been adequately measured (though not yet at the desired level of precision).

Inclusion of nonthermal emission due to Compton scattering of relativistic electrons by the FIR and CMB fields is an important new ingredient in the modeling of the high energy emission in an evolving population of SBGs. Although the level of this emission is still unknown, our explicit accounting for this emission and its expected rapid rise with redshift (which makes its relative contribution very significant at $z>1$ ) provide further motivation for a more detailed description than given here. (The occurrence of low-luminosity AGNs in the nuclei of SBGs is a controversial issue (e.g., Veilleux 2001), so we chose not to include such a component in the current treatment.)

Our main result that the evolving population of SBGs contributes appreciably to the $\mathrm{CXB}$ in the $2-10 \mathrm{keV}$ band is quite consistent with our previous estimates (Rephaeli et al. 1991, 1995), and a similar result by Griffiths \& Padovani (1990). It was argued in the latter paper that since the spectrum of $\mathrm{X}$ ray binaries is quite hard and comparable in shape to that of the residual CXB in the $2-30 \mathrm{keV}$ range, evolving SBGs in the redshift range $0.5 \lesssim z \lesssim 1$ could make an important contribution to the $2-10 \mathrm{keV} \mathrm{CXB}$. In the use of a quantitative spectral form and evolutionary function, our treatment is similar in spirit to that of Ricker \& Meszaros (1993, hereafter RM93). The main improvement with respect to the work of RM93 is the use of spectral results from individual SBGs observed with $A S C A$ and BeppoSAX and the inclusion of the two Compton components, in contrast to their use of essentially an assumed theoretical model. (We have included also the observed warm $(\simeq 0.8 \mathrm{keV})$ component, but its contribution to the emission at $\epsilon>2 \mathrm{keV}$ is relatively minor.)

In order to check the stability of our results vs. the characteristics of the FIR-selected SBG population, we have repeated the calculation using the SBG population parameters of Franceschini et al. (2001), who fitted the $12 \mu \mathrm{m}$ number counts invoking the presence of a starbursting population whose volume emissivity increases steeply out to $z=0.8$, and remains constant at higher $z$ (in a flat $\Lambda\left(\Omega_{\mathrm{m}}=0.3, \Omega_{\Lambda}=0.7\right)$ cosmological model). The contribution of Franceschini et al.'s
SBG population ${ }^{8}$ turns out to be only marginally higher than that computed for the SBG population of Pearson \& Rowan-Robinson (1996) used in our main calculation and described by Eqs. (2)-(5) (with $q=3$ ). On the whole, then, the results of this variant calculation are qualitatively consistent with our main results (see Fig. 5, where the "wind+binaries" spectral model is used).

Recent results from the Chandra deep surveys provide direct estimates of the SBG contribution to the CXB. Using the 1 Ms Chandra Deep Field North (Brandt et al. 2001a) and $15 \mu \mathrm{m}$ ISOCAM Hubble Deep Field North (Aussel et al. 1999) surveys, Alexander et al. (2002) found a tight correlation between the population of strongly evolving SBGs at $z \sim 1$ discovered in faint $15 \mu \mathrm{m}$ ISOCAM surveys (e.g., Aussel et al. 1999; Elbaz et al. 1999, 2002) - whose space density is an order of magnitude higher than that of local SBGs - and the apparently normal galaxies detected at faint fluxes in X-ray surveys (Giacconi et al. 2001; Hornschemeier et al. 2001; Brandt et al. 2001b). The characteristics $\left(L_{\mathrm{FIR}}, L_{x}, 2-10 \mathrm{keV}\right.$ spectral slope) of the population of the X-ray detected galaxies, whose redshifts are in the range $0.4 \leq z \leq 1.3$ (based mainly on Keck data; see Alexander et al. 2002 and references therein), are consistent with those expected for SBGs resembling local objects such as M 82 and NGC 3256. In particular, their stacked average $2-10 \mathrm{keV}$ spectral slope, $\Gamma \sim 2$, is steep enough to suggest a low fraction of obscured AGN activity within the population. The contribution of this population to the $0.5-8 \mathrm{keV}$ CXB, estimated to be $\sim 2 \%$ (Alexander et al. 2002), marginally agrees (on the low side) with our result for $q=3$ (see Fig. 4). A contribution to the CXB will also come from the $1 \mathrm{Ms}$ Chandra Deep Field North sources identified as Lyman-break galaxies at $z \sim 2-4$ : these star-forming sources have rest-frame $2-8 \mathrm{keV}$ luminosities and $\mathrm{X}$-ray/ $B$-band luminosity ratios that are comparable to those of local SBGs (Brandt et al. 2001c). Based on the above, there is no evidence for a substantial change in the $\sim 2-10 \mathrm{keV}$ luminosity and spectral slope of these Xray-selected, star-forming galaxies. On the other hand, their IR (15 $\mu \mathrm{m}$ ISOCAM) counterparts do show a strong density evolution with redshift (e.g., Aussel et al. 1999; Elbaz et al. 1999, 2002). All this clearly supports our adopted scheme of cosmic evolution, which is essentially of the density type (see Sect. 4).

Finally, it is commonly thought that AGNs are the prime sources of the CXB (e.g.: Comastri et al. 1995; Setti \& Woltjer 1989; Boldt \& Leiter 1981; Leiter \& Boldt 1982), and that additional contributions from other sources are typically estimated to be $\$ 20 \%$ in the $2-10 \mathrm{keV}$ range (Giacconi et al. 2001; Tozzi et al. 2001; Mushotzky et al. 2000). Based on our results, SBGs may possibly be the second most important

\footnotetext{
8 The local 2-10 luminosity density of the starbursting population of Franceschini et al. (2001) is $\mathcal{L}(0)=3.15 \times 10^{36} \mathrm{erg} \mathrm{s}^{-1} \mathrm{Mpc}^{-1}$. This value is deduced from the $12 \mu \mathrm{m}$ local luminosity function of this population (see their Fig. 9), which can be described as $\phi(L) \mathrm{d} \log L=\phi_{\star} L^{1-a}\left[1+L /\left(b L_{\star}\right)\right]^{-b} \mathrm{~d} \log L$ with $\phi_{\star}=5.5 \times 10^{-3}$, $L_{\star}=5 \times 10^{9} L_{\odot}, a=1.15$, and $b=3.1$, and the average $2-10 \mathrm{keV}$ to $12 \mu \mathrm{m}$ luminosity ratio of $(1.26 \pm 0.35) \times 10^{-3}$, derived for the same sample of local SBGs mentioned in footnote 3 (the $12 \mu \mathrm{m}$ data are from Shapley et al. 2001). The evolution is $\mathcal{L}(z)=\mathcal{L}(0)(1+z)^{7.8}$ for $z \leq z_{\text {break }}$ and $\mathcal{L}(z)=\mathcal{L}(0)\left(1+z_{\text {break }}\right)^{7.8}$ for $z>z_{\text {break }}$, with $z_{\text {break }}=0.8$.
} 

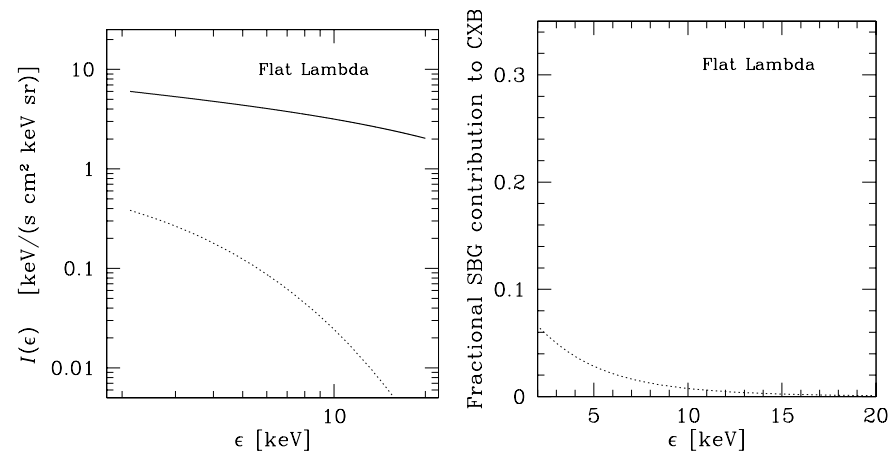

Fig. 5. The spectrum of the integrated background light (left), and the corresponding contribution to the CXB (right), arising from the SBG population of Franceschini et al. (2001), assuming a "starburst only" model for the spectrum. See the text for more details.

contributors to the CXB. (Clusters of galaxies also contribute a few percent: e.g., Piccinotti et al. 1982.) The importance of determining the level of the SBG contribution to the $2-10 \mathrm{keV}$ CXB lies not only in the implications for the evolution of SBGs themselves, but also in the ramifications for galaxy evolution in general, and as an additional input on the evolution of the AGN phenomenon in particular (e.g.: Fabian et al. 1998; Gilli et al. 1999).

Acknowledgements. We are glad to thank Elihu Boldt for enlightening exchanges on the CXB normalization. We also thank the $n$ anonymous referee for useful suggestions. M.P. acknowledges financial support from the Italian Space Agency (ASI) through grant No. ASI RS 99 $\mathrm{I} / \mathrm{R} / 098 / 00$, as well as the warm and stimulating environment of the Center for Astrophysics and Space Sciences (CASS) of the University of California at San Diego (UCSD), where part of this work was carried out. Y.R. acknowledges NASA for supporting his work at UCSD. This research has made use of the NASA/IPAC Extragalactic Database (NED) which is operated by the Jet Propulsion Laboratory, California Institute of Technology, under contract with NASA.

\section{References}

Alexander, D. M., Aussel, H., Bauer, F. E., et al. 2002, ApJ, 568, L85 Aussel, H., Cesarsky, C. J., Ebaz, D., \& Starck, J. L. 1999, A\&A, 342, 313

Benn, C. R., Rowan-Robinson, M., McMahon, R. G., Broadhurst, T. J., \& Lawrence, A. 1993, MNRAS, 263, 98

Boldt, E. 1987, Phys. Rep., 146, 215

Boldt, E., \& Leiter, D. 1981, Nature, 290, 483

Bookbinder, J., Cowie, L. L., Krolik, J. H., \& Rees, M. 1980, ApJ, 237, 647

Brandt, W. N., Alexander, D. M., Hornschemeier, A. E., et al. 2001a, AJ, 122, 2810

Brandt, W. N., Hornschemeier, A. E., Alexander, D. M., et al. 2001b, AJ, 122, 1

Brandt, W. N., Hornschemeier, A. E., Schneider, D. P., et al. 2001c, ApJ, 558, L5

Cappi, M., Persic, M., Bassani, L., et al. 1999, A\&A, 350, 777

Chen, L.-W., Fabian, A. C., \& Gendreau, K. C. 1997, MNRAS, 285, 449

Christian, D. J, \& Swank, J. H. 1997, ApJS, 109, 177
Comastri, A., Setti, G., Zamorani, G., \& Hasinger, G. 1995, A\&A, 296,1

Condon, J. J. 1992, ARA\&A, 30, 575

Dahlem, M., Weaver, K. A., \& Heckman, T. M. 1998, ApJS, 118, 401

Danese, L., De Zotti, G., Franceschini, A., \& Toffolatti, L. 1987, ApJ, 318, L15

David, L. P., Jones, C., \& Forman, W. 1992, ApJ, 338, 82

Della Ceca, R., Griffiths, R. E., Heckman, T. M., Lehnert, M. D., \& Weaver, K. A. 1999, ApJ, 514, 772

Della Ceca, R., Griffiths, R. E., Heckman, T. M., \& MacKenty, J. W. 1996, ApJ, 469, 662

Elbaz, D., Cesarsky, C. J., Chanial, P., et al. 2002, A\&A, 384, 848

Elbaz, D., Cesarsky, C. J., Fadda, D., et al. 1999, A\&A, 351, L37

Ellis, R. S., Colless, M., Broadhurst, T., Heyl, J., \& Glazebrook, K. 1996, MNRAS, 280, 235

Fabbiano, G. 1989, ARA\&A, 27, 87

Fabian, A. C., Barcons, X., Almaini, O., \& Iwasawa, K. 1998, MNRAS, 297, L11

Franceschini, A., Aussel, H., Cesarsky, C. J., Elbaz, D., \& Fadda, D. 2001, A\&A, 378, 1

Franceschini, A., Danese, L., De Zotti, G., \& Xu, C. 1988, MNRAS, 233,175

Franceschini, A., Braito, V., Persic, M., et al. 2002, MNRAS, submitted

Fried, J. W., von Kuhlmann, B., Meisenheimer, K., et al. 2001, A\&A, 367,788

Gendreau, K. C., Mushotzky, R., Fabian, A.C., et al. 1995, PASJ, 47, L5

Giacconi, R., Rosati, P., Tozzi, P., et al. 2001, ApJ, 551, 624

Gilli, R., Risaliti, G., \& Salvati, M. 1999, A\&A, 347, 424

Goldshmidt, O., \& Rephaeli, Y. 1995, ApJ, 444, 113

Griffiths, R., \& Padovani, P. 1990, ApJ, 360, 483

Griffiths, R. E., Ptak, A., Feigelson, E. D., et al. 2000, Science, 290, 1325

Gruber, D. E., Matteson, J. L., Peterson, L. E., \& Jung, G. V. 1999, ApJ, 520, 124

Hornschemeier, A. E., Brandt, W. N., Garmire, G. P., et al. 2001, ApJ, 554,742

Itoh, N., Sakamoto, T., Kusano, S., Nozawa, S., \& Koyama, Y. 2000, ApJS, 128, 125

Kim, D.-C., \& Sanders, D. B. 1998a, ApJ, 508, 627

Kim, D.-C., \& Sanders, D. B. 1998b, ApJS, 119, 41

Leiter, D., \& Boldt, E. 1982, ApJ, 260, 1

Lilly, S. J., Le Fevre, O., Hammer, F., \& Crampton, D. 1996, ApJ, 460, L1

Lilly, S. J., Tresse, L., Hammer, F., Crampton, D., \& Le Fevre, O. 1995, ApJ, 455, 108

Lin, H., Yee, H. K. C., Carlberg, R. G., et al. 1999, ApJ, 518, 523

Lonsdale, C. J., Hacking, P. B., Conrow, T. P., \& Rowan-Robinson, M. 1990, ApJ, 358, 60

Lonsdale, C. J., Helou, G., Good, J. C., \& Rice, W. 1985, Cataloged Galaxies and Quasars Observed in the IRAS Survey (JPL, Pasadena, CA)

Madau, P., Ferguson, H. C., Dickinson, M. E., et al. 1996, MNRAS, 283, 1388

Marshall, F. E., Boldt, E. A., Holt, S. S., et al. 1980, ApJ, 235, 4

Matsumoto, H., \& Tsuru, T. G. 1999, PASJ, 51, 321

Miyaji, T., Ishisaki, Y., Ogasaka, Y., et al. 1998, A\&A, 334, L13

Mizuno, T., Ohbayashi, H., Iyomoto, N., \& Makishima, K. 1998, in The Hot Universe, ed. K. Koyama, S. Kitamoto, \& M. Itoh, IAU Symp., 188, 284

Moran, E. C., Lehnert, M. D., \& Helfand, D. J. 1999, ApJ, 526, 649

Morrison, R., \& McCammon, D. 1983, ApJ, 270, 119 
Mushotzky, R. F., Cowie, L. L., Barger, A. J., \& Arnaud, K. A. 2000, Nature, 404, 459

Okada, K., Mitsuda, K., \& Dotani, T. 1997, PASJ, 49, 653

Pearson, C., \& Rowan-Robinson, M. 1996, MNRAS, 283, 174

Persic, M., Gruber, D. E., \& Rephaeli, Y. 2002, in preparation

Persic, M., \& Rephaeli, Y. 2002, A\&A, 382, 843

Piccinotti, G., Mushotzky, R. F., Boldt, E. A., et al. 1982, ApJ, 253, 485

Ptak, A., Serlemitsos, P. J., Yaqoob, T., Mushotzky, R., \& Tsuru, T. 1997, AJ, 113, 1286

Rephaeli, Y. 1979, ApJ, 227, 364

Rephaeli, Y., \& Gruber, D. 2002, A\&A, 389, 752

Rephaeli, Y., Gruber, D., \& Persic, M. 1995, A\&A, 300, 91

Rephaeli, Y., Gruber, D., Persic, M., \& McDonald, D. 1991, ApJ, 380, L59

Ricker, P. M., \& Meszaros, P. 1993, ApJ, 418, 49

Roberts, T. P., Schurch, N. J., \& Warwick, R. S. 2001, MNRAS, 324, 737

Rothschild, R. E., Baity, W. A., Gruber, D. E., et al. 1983, ApJ, 269, 423

Rowan-Robinson, M., \& Crawford, J. 1989, MNRAS, 238, 523

Rowan-Robinson, M., Benn, C. R., Lawrence, A., McMahon, R. G., \& Broadhurst, T. J. 1993, MNRAS, 263, 123

Saunders, W., Rowan-Robinson, M., Lawrence, A., et al. 1990, MNRAS, 242, 318
Schaaf, R., Pietsch, W., Biermann, P. L., Kronberg, P. P., \& Schmutzler, T. 1989, ApJ, 336, 722

Schmitt, H. R., Kinney, A. L., Calzetti, D., \& Storchi Bergmann, T. 1997, AJ, 114, 592

Setti, G., \& Woltjer, L. 1989, A\&A, 224, L21

Shapley, A., Fabbiano, G., \& Eskridge, P. B. 2001, ApJS, 137, 139

Silva, L., Granato, G. L., Bressan, A., \& Danese, L. 1998, ApJ, 509, 103

Stewart, G. C., Fabian, A. C., Terlevich, R. J., \& Hazard, C. 1982, MNRAS, 200, 61P

Thompson, R. I., Weymann, R. J., \& Storrie-Lombardi, L. J. 2001, ApJ, 546, 694

Tozzi, P., Rosati, P., Nonino, M., et al. 2001, ApJ, 562, 42

Tucker, W. A. 1975, Radiation Processes in Astrophysics (Cambridge: MIT Press), 169

Veilleux, S. 2001, in Starburst Galaxies: Near and Far, ed. L.Tacconi, \& D. Lutz (Heidelberg: Springer-Verlag), 88

Weedman, D. W. 1987, in Star Formation in Galaxies, ed. C. J. Lonsdale (NASA CP-2466), 351

White, N. E., Swank, J. H., \& Holt, S. S. 1983, ApJ, 270, 711

Wilson, G., Cowie, L., Barger, A. J., \& Burke, D. J. 2002, AJ, 124, 1258

Zezas, A. L., Georgantopoulos, I., \& Ward, M. J. 1998, MNRAS, 301, 915 